\title{
Solar cycle effect on geomagnetic storms caused by interplanetary magnetic clouds
}

\author{
C.-C. $\mathbf{W u}^{1,2,3}$ and R. P. Lepping ${ }^{3}$ \\ ${ }^{1}$ CSPAR/The University of Alabama in Huntsville, Huntsville, AL 35899, USA \\ ${ }^{2}$ also at: Key State of Laboratory for Space Weather, Chinese Academy of Sciences, Beijing 100080, China \\ ${ }^{3}$ Heliophysics Science Division, NASA/GSFC, Greenbelt, MD 20771, USA
}

Received: 4 April 2006 - Revised: 9 October 2006 - Accepted: 13 October 2006 - Published: 21 December 2006

\begin{abstract}
We investigated geomagnetic activity which was induced by interplanetary magnetic clouds during the past four solar cycles, 1965-1998. We have found that the intensity of such geomagnetic storms is more severe in solar maximum than in solar minimum. In addition, we affirm that the average solar wind speed of magnetic clouds is faster in solar maximum than in solar minimum. In this study, we find that solar activity level plays a major role on the intensity of geomagnetic storms. In particular, some new statistical results are found and listed as follows. (1) The intensity of a geomagnetic storm in a solar active period is stronger than in a solar quiet period. (2) The magnitude of negative $B z_{\min }$ is larger in a solar active period than in a quiet period. (3) Solar wind speed in an active period is faster than in a quiet period. (4) $V B s_{\max }$ in an active period is much larger than in a quiet period. (5) Solar wind parameters, $B z_{\min }, V_{\max }$ and $V B s_{\max }$ are correlated well with geomagnetic storm intensity, $D_{s t_{\min }}$ during a solar active period. (6) Solar wind parameters, $B z_{\min }$, and $V B s_{\max }$ are not correlated well (very poorly for $V_{\max }$ ) with geomagnetic storm intensity during a solar quiet period. (7) The speed of the solar wind plays a key role in the correlation of solar wind parameters vs. the intensity of a geomagnetic storm. (8) More severe storms with $D_{s t_{\min }} \leq-100 \mathrm{nT}$ caused by MCs occurred in the solar active period than in the solar quiet period.
\end{abstract}

Keywords. Interplanetary physics (Interplanetary magnetic fields; Solar wind plasma) - Magnetospheric physics (Storms and substorms)

\section{Introduction}

Many scientists have tried to understand the fundamental mechanisms that explain the geomagnetic storm since storm

Correspondence to: C.-C. Wu

(wuc@cspar.uah.edu) patterns were explored by Chapman (1919). The primary cause of a magnetic storm is long duration, intense southward interplanetary magnetic fields which interconnect with the Earth's magnetic field and allow solar wind energy transport into the Earth's magnetosphere (e.g. Tsurutani et al., 1988; Gonzalez et al., 1994). Magnetic clouds (Mcs) are major sources of long-lasting strong southward interplanetary magnetic field (IMF) $B_{z}$ and, therefore, usually cause magnetic storms (Burlaga et al., 1981; Wilson, 1990). However, the largest (in duration) Mcs do not necessarily have the most intense field strengths (Farrugia et al., 1997). Nor are the most intense (strong $|B|$ ) clouds the most geoeffective, especially if the MC of interest is highly inclined with respect to the ecliptic plane and possesses a positive axial field. Then there will be little or no negative $B_{z}$ (Lepping et al., 2003). A geomagnetic storm might be driven by a MC itself or by the sheath fields upstream of a MC if an upstream exists (e.g., Lepping and Berdichevsky, 2001; Wu and Lepping, 2002a).

Various changes in the IMF are well known to play a key role in regulating geomagnetic activity (e.g., Fairfield and Cahill, 1966). In particular, the variation of the north-south component of the IMF $\left(B_{z}\right)$, when rendered in the geocentric solar-magnetospheric (GSM) coordinate system, plays a crucial role in determining the amount of solar wind energy that is transferred to the magnetosphere (e.g. Arnoldy, 1971). Using Explorer 12 measurements of the magnetic field outside the magnetosphere and comparing with ground magnetograms from arctic observatories, Fairfield and Cahill (1966) showed that an IMF with a southward component tends to be associated with a ground disturbance, whereas a northward IMF is associated with a quiet condition. The early studies of "solar wind-magnetosphere coupling" have concentrated on a single coupling function (e.g. Perreault and Akasofu, 1978; Kan and Lee, 1979; Doyle and Burke, 1983; Gonzalez et al., 1989) which contains either a single or several solar wind parameters (e.g. Gonzalez et al., 1994).

Published by Copernicus GmbH on behalf of the European Geosciences Union. 

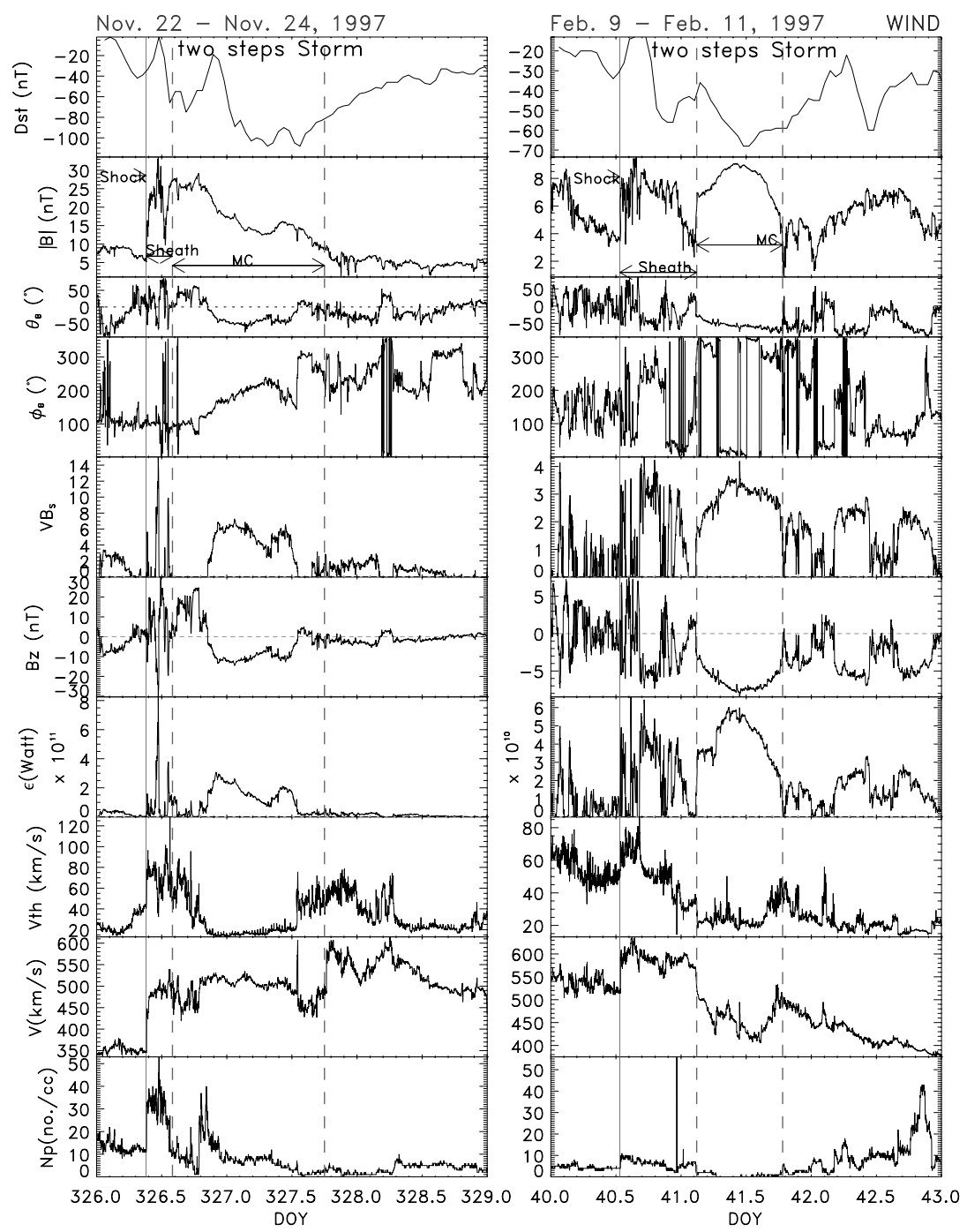

Fig. 1. Solar wind parameters and $D s t$ for two magnetic clouds: 22-24 November 1997 and 9-11 February 1997. The parameters are (from top to bottom): geomagnetic index $\left(D_{s t}\right)$, interplanetary magnetic field magnitude $(|B|)$, field latitude $\left(\theta_{B}\right)$, and longitude $\left(\phi_{B}\right)$, induced electric field $\left(V B_{S}\right)$, north-south component of the IMF $\left(B_{z}\right)$, Akasofu (1981) $\epsilon$, proton thermal speed $\left(V_{t h}\right)$, plasma bulk speed $(V)$, proton density $\left(N_{P}\right)$.

Burton et al. (1975) presented a formula for predicting the $D_{s t}$ index from knowledge of the velocity and density of the solar wind and the north-south component of the interplanetary magnetic field (in GSM coordinates). The intensity of a magnetic storm which is associated with a geomagnetic cloud is predictable (Wu and Lepping, 2005).

$\mathrm{Wu}$ and Lepping (2002a) used the first four years of WIND magnetic field (Lepping et al., 1995) and plasma data (Ogilvie et al., 1995) to investigate the relationships between solar wind parameters and geomagnetic storms. They found that the intensity of geomagnetic storms $\left(D_{s t_{\text {min }}}\right)$ is strongly related to the magnitude of solar wind parameters $\left(B_{z}, V B_{S}\right.$ or $\epsilon$ in Akasofu, 1981) within magnetic cloud complexes (including upstream sheath regions), and the occurrence tim- ing of a geomagnetic storm is associated with the occurrence timing of $B z_{\min },(V B s)_{\max }$ or $\epsilon_{\max } . D_{s t_{\min }}$ is minimum $D_{s t}$, $B z_{\text {min }}$ is minimum $B_{z},(V B s)_{\max }$ is maximum $V B s$, and $\epsilon_{\max }$ is the maximum $\epsilon$ (Akasofu, 1981) value observed during or in front of a MC event (i.e., in the sheath). (The word "event" here usually means the entire "sheath"/cloud complex.) $B_{s}$ is the southward component of the IMF $\left(B_{s}=\left|B_{z}\right|\right.$ for $B_{z}<0$ and $B_{s}=0$ for $B_{z} \geq 0$ ). Using the solar wind parameters of the apparent 135 magnetic clouds observed between 1965 and 1998 from the OMNI data set, Wu and Lepping (2002b) found that storm intensity $\left(D_{s t_{\min }}\right)$ is strongly related to the IMF in the $z$-direction $\left(B_{z}\right)$ and to $V B s$, but not well correlated with solar wind velocity alone. However, the relationship between storm intensity and $\mathrm{Bz}$ (or 


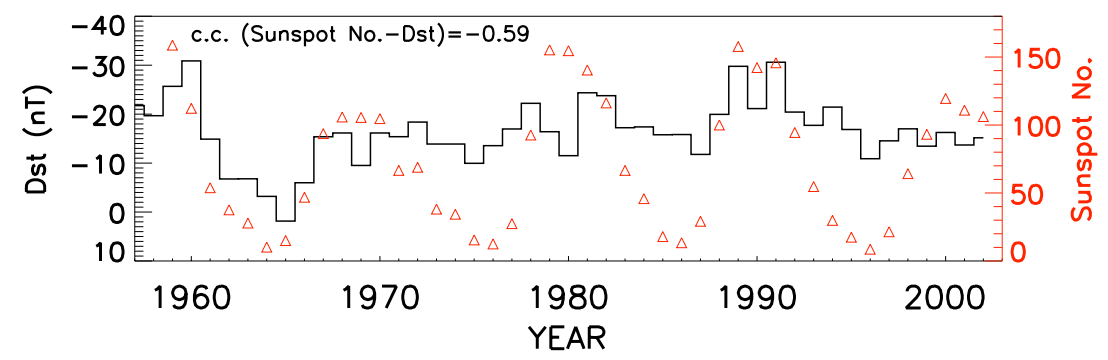

Fig. 2. Yearly averages of sunspot number $(\triangle)$ and geomagnetic activity index, $D_{s t}$ (solid line), during 1957-2002.

$V B s$ ) shows better correlation as the solar wind velocity gets faster. Eighty-two interplanetary magnetic clouds were identified by the MFI team by using WIND solar wind plasma and magnetic field data from solar minimum through the maximum of solar cycle 23 (1995-2003) (Lepping et al., 2006). The occurrence frequency of MCs appears to be related neither to the occurrence of coronal mass ejections as observed by SOHO LASCO nor to the sunspot number (e.g. Wu et al., 2003, 2006). In contrast, the intensity of geomagnetic storms related to magnetic clouds is affected by both solar activity and the occurrence frequency of CMEs, and more than $\sim 87 \%$ of MCs induced geomagnetic storms (Wu et al., 2003, 2006). Wu et al. (2006) also found that the occurrence of magnetic cloud-like structures (MCLs) or the occurrence rate of the joint set (MCs+MCLs) are correlated with both sunspot number and the occurrence rate of CMEs. This motivated us to study several relationships for solar activity vs. various solar wind parameters in order to understand the solar cycle effect on geomagnetic storms which is caused by MCs.

In Sect. 2 we present associated data analysis, storm event selection and the derived storm intensities, $D_{s t_{\min }}$, and prediction schemes, which include both velocity dependence and independence. The results will be discussed in Sect. 3, and some conclusions are stated in Sect. 4.

\section{Data analysis}

Four data sets are used in this study. The first data set, OMNI solar wind plasma and magnetic field data, which were obtained from NSSDC/NASA-GSFC, were used for most earlier events. The second data set, WIND solar wind plasma and magnetic field data, were obtained from the WIND SWE and MFI groups for the events after 1995. Both the third data set (the geomagnetic activity index, $D_{s t}$ ) and the forth data set (sunspot number) were obtained from National Geophysical Data Center, Boulder, Colorado, USA.

\subsection{Magnetic cloud event selection}

The MC events used in the present study have been extracted from three previously published reports (Klein and Burlaga,
1982; Bothmer and Rust, 1997; Lepping and Berdichevsky, 2000). Hourly averages of solar plasma and magnetic field data from OMNI and $D_{s t}$ during these cloud events were compiled into a data base. Thus, a total of 135 events which cover the period from 1965 to 1998 were obtained for this analysis. (It should be understood that this set of 135 events must be considered a subset of the full set of MCs occurring over the period 1965-1998, since there were many data gaps due to spacecraft tracking limitations and from times when the spacecraft were behind the bowl shock.) This data set has been used to study the effect of solar wind speed on magnetic cloud-associated magnetic storm intensity ( $\mathrm{Wu}$ and Lepping, 2002b).

Figure 1 shows two examples of MCs which occurred during 22-24 November and 9-11 February 1997. Note that each MC produced a two-step (two main phases) geomagnetic storm. In Fig. 1, we define the following: $\epsilon_{\max }$ is the maximum value of the Akasofu [1981] $\epsilon$ observed during a cloud event. Note that $D_{s t_{\min }}$ is the minimum value of $D_{s t} \mathrm{ob}-$ served during a cloud "event" (where "event" here and below usually means the entire "sheath"/cloud complex.), $V B s_{\max }$ is the maximum value of $V B s$ observed during a cloud event, where $B s$ is the southward component of the $\operatorname{IMF}\left(B s=\left|B_{z}\right|\right.$ for $B_{z}<0$ and $B s=0$ for $\left.B_{z} \geq 0\right) ; B z_{\min }$ is minimum $B_{z}$ value observed during or in front of a MC event (i.e., in the sheath region, if there is an upstream shock).

The yearly averages of sunspot number and geomagnetic activity index $\left(D_{s t}\right)$ for the years 1965-2002 are shown in Fig. 2. The solid line represents yearly averaged $D_{s t}$ and the triangles represent yearly averaged sunspot number. The correlation coefficient (c.c.) between yearly sunspot number and $D_{s t}$ is -0.59 . Figure 2 also shows that solar minima occurred in 1964, 1976, 1986 and 1996 and solar maxima occurred in 1969, 1980, 1991 and 2000.

Figure 3 shows plots of $D_{s t_{\min }}$ vs. $\left|B z_{\min }\right|, V_{\max }$ and $V B s_{\max }$ with respect to $D_{s t}$ and resulting correlation coefficients for the solar active periods (years of 1968, 1969, 1979, $1980,1981,1990,1991,1992$ ) and quiet periods (years of 1965, 1966, 1975, 1976, 1985, 1986, 1996, 1997). There were $32 \mathrm{MCs}$ observed during the active period and $33 \mathrm{MCs}$ observed during the quiet period. Table 1 summarizes the correlation coefficients of $D_{s t_{\min }}$ vs. three different solar wind 

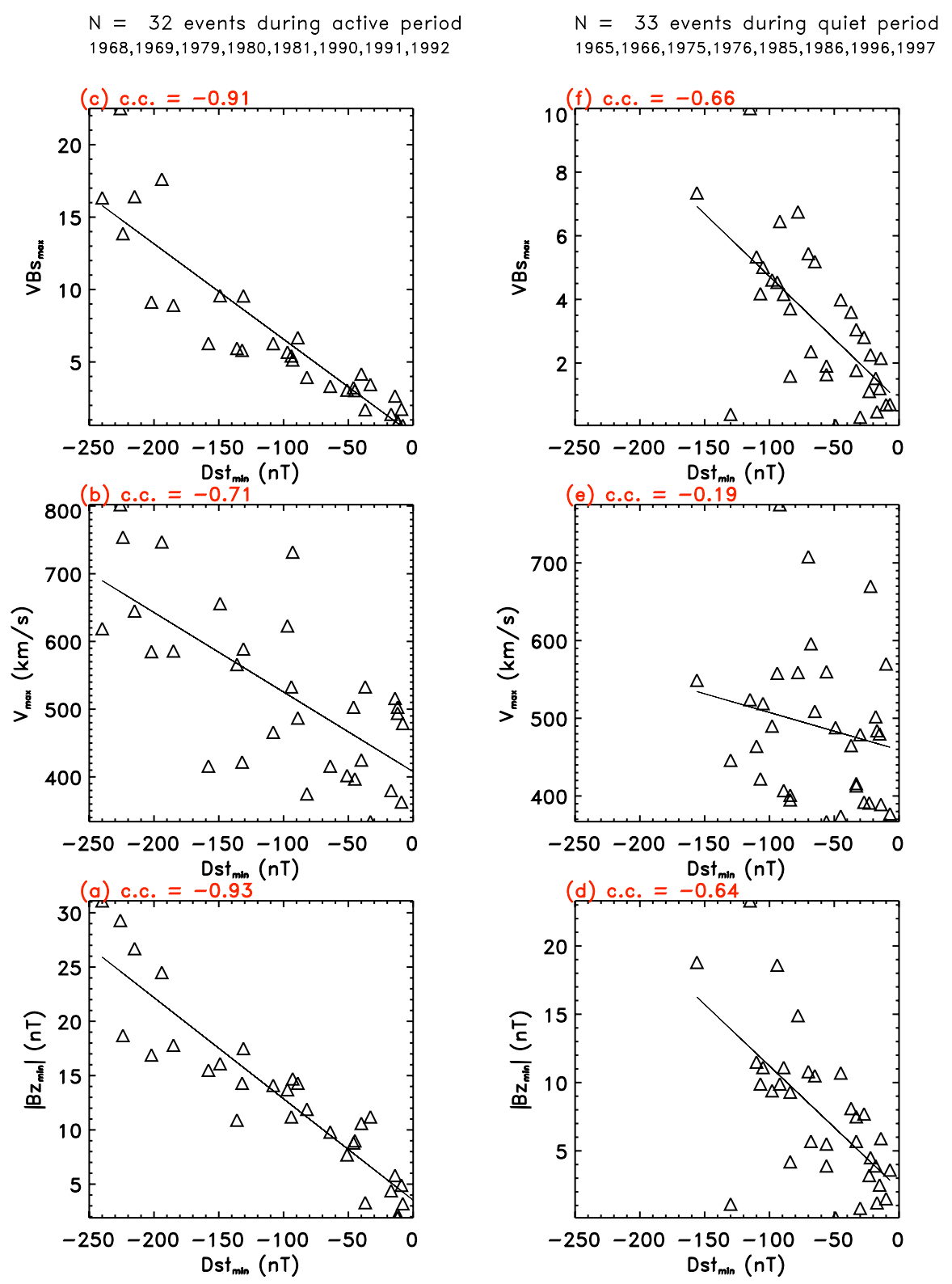

Fig. 3. Relationships and correlation coefficients (c.c.) for storm intensity $\left(D_{s t}\right)$ vs. various solar wind parameters (see the text). The left column represents the results for the 32 events the during solar active period; the right column represents the results for the 33 events during solar quiet period.

parameters. It shows clearly that all three solar wind parameters are well correlated with $D_{s t_{\min }}$ during solar maximum but have a poor correlation in solar minimum. For example, the c.c. is quite high $(-0.93)$ between $\left|B z_{\min }\right|$ and $D_{s t_{\min }}$ for the active period.

Figures 4 and 5 show the histograms of $V_{\max }, D_{s t_{\min }}$, $B z_{\min }$ and $V B s_{\max }$ for both solar quiet and active periods, respectively. Table 2 summarizes the results related to Figs. 4 and 5. Both figures show that: (1) the solar wind speed is faster in solar maximum than in solar minimum; (2) $|B z|$ at $B z_{\min }$ is greater in solar maximum than in solar minimum;
(3) the averaged storm intensity, $\left\langle D_{s t_{\min }}>\right.$ is more intense in solar maximum than the averaged $<D_{s t_{\text {min }}}>$ in solar minimum; and (4) averaged $V B s_{\max }$ in solar maximum is two times larger than what it is in solar minimum.

Table 3 summarizes the occurrence frequency of MCs which caused different strengths of geomagnetic storms during both solar quiet and active periods. More than twice as many MCs causing severe geomagnetic storms $\left(D_{s t_{\min }} \leq-\right.$ $100 \mathrm{nT}$ ) occurred in the solar active period (13 MCs) as in quiet period (6 MCs). 
Table 1. Summarized correlation coefficient for $D_{s t_{\min }}$ vs. $\left|B z_{\min }\right|$, $V_{\max }$ and $V B s_{\max }$.

\begin{tabular}{lccc}
\hline & $\begin{array}{c}\left|B z_{\min }\right| \\
(\mathrm{nT})\end{array}$ & $\begin{array}{c}V_{\max } \\
(\mathrm{km} / \mathrm{s})\end{array}$ & $V B s_{\max }$ \\
\hline$D_{s t_{\min }}$ (active period) $^{\mathrm{a}}$ & -0.93 & -0.71 & -0.91 \\
$D_{s t_{\min }}$ (quiet period) $^{\mathrm{b}}$ & -0.64 & -0.19 & -0.66 \\
\hline
\end{tabular}

a Events occurred during the years 1968, 1969, 1979, 1980, 1981, 1990, 1991 and 1992.

b Events occurred during the years 1965, 1966, 1975, 1976, 1985, 1986, 1996, and 1997.

\section{Discussion}

Using 34 MCs that occurred during 1995-1998, Wu and Lepping (2002a) found that the correlation between storm intensity $\left(D_{s t_{\min }}\right)$ and the z-component of the interplanetary magnetic field, IMF $(B z)$, (or with $V B s$ ) is very high. For solar cycle 23 , previous studies have shown that the yearly average geomagnetic activity index $\left(D_{s t}\right)$ is not correlated well with solar activity (Wu et al., 2003), but the intensity of geomagnetic storms induced by MCs (observed during solar cycle 23) is related to solar activity (Wu et al., 2003, 2006). (The correlation coefficient for the yearly averages of sunspot number vs. yearly averages of intensity of magnetic storms due to MCs is 0.83 (Wu et al., 2006).) Using data from 4 solar cycles, the results of this study show that the correlation coefficient is poor (c.c. $=-0.59$ ) between the yearly average geomagnetic activity index and sunspot number. This result reconfirms the reports of previous studies.

Cane et al. (2000) studied the relationship between the intensity of geomagnetic storms (with $D_{s t_{\min }}<-50 \mathrm{nT}$ ) and southward interplanetary magnetic field strength in ejecta or sheath regions for events associated with front-side halo CMEs during 1996-1999. They found that storm intensity versus the maximum southward magnetic field $(B s)$ in either the ejecta or the adjacent disturbed solar wind has a correlation coefficient of -0.74 . Using $34 \mathrm{MCs}$ observed between 1995 and 1998, Wu and Lepping (2002a) found that the $D_{s t_{\min }}$ is strongly correlated with $V B s_{\max }$ and $\left|B z_{\min }\right|$ with c.c. $=-0.79$ and -0.77 , respectively. Both of the earlier studies (Cane et al., 2000; Wu and Lepping, 2002a) show that the intensity of a storm is strongly correlated with the magnitude of $B_{z}$. However, both studies cover less than $1 / 4$ of solar activity cycle (where 22 years is a full solar cycle). The correlation coefficient of $\left|B z_{\min }\right|$ and $D_{s t_{\min }}$ is similar for both of these earlier studies (i.e., -0.74 for Cane et al. and -0.77 for $\mathrm{Wu}$ and Lepping). Using $135 \mathrm{MCs}$ which occurred during 1965-1998 (33 years, one and a half full solar cycles), Wu and Lepping (2002b) found that the intensity of a geomagnetic storm is primarily related to the solar wind parameters $B_{z}$ and $V B s$. The results confirmed the two ear-
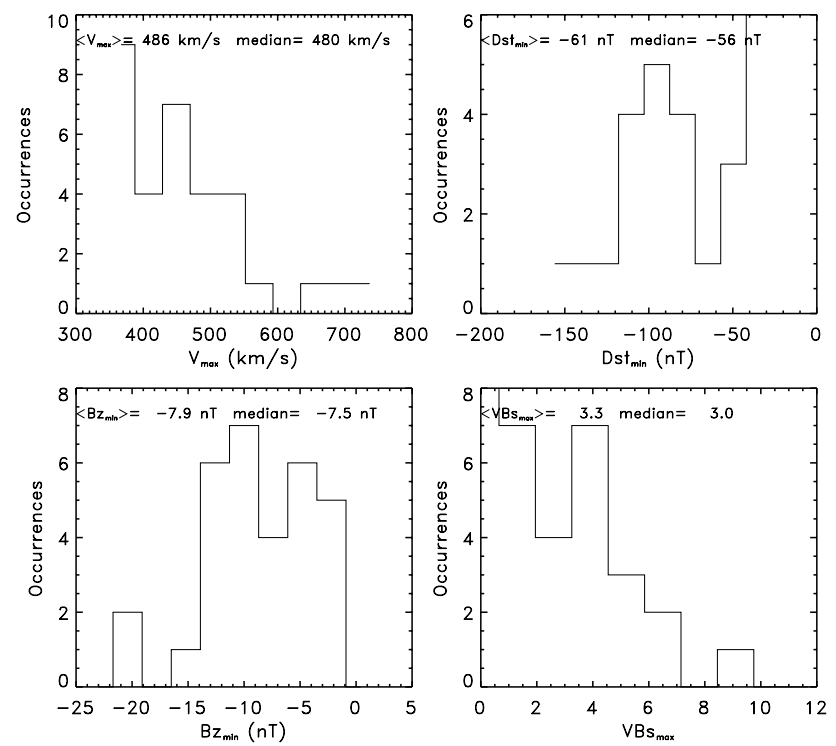

Fig. 4. Histograms of $V_{\max }, D_{s t_{\min }}, B z_{\min }$ and $V B s_{\max }$ for MCs during the quiet time of the solar cycle for the years 1965, 1966, 1975, 1976, 1985, 1986, 1996, 1997.
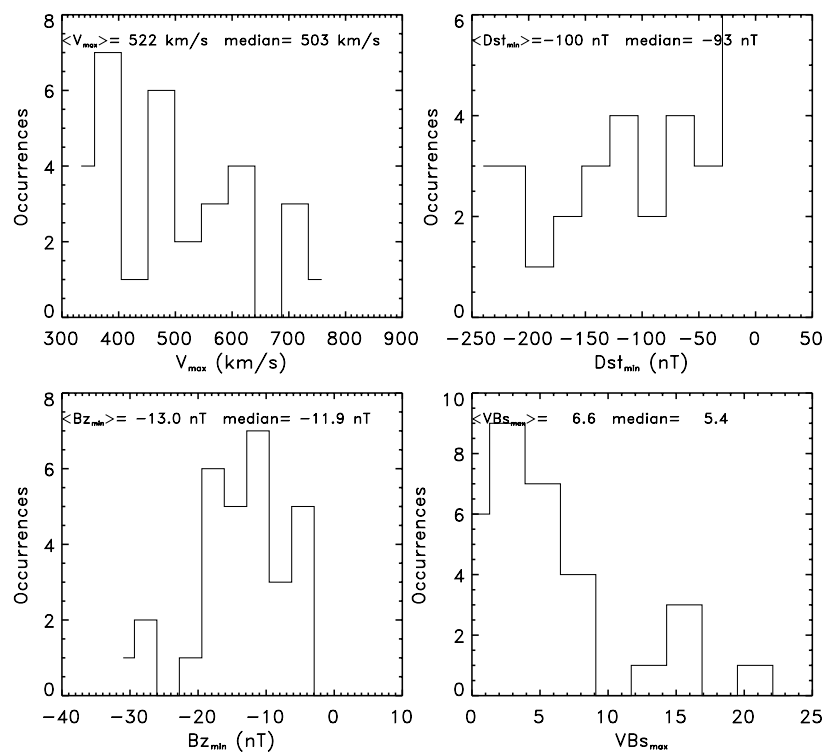

Fig. 5. Histograms of $V_{\max }, D_{s t_{\min }}, B z_{\min }$ and $V B s_{\max }$ for MCs during the active time of the solar cycle for the years of 1968,1969 , 1979, 1980, 1981, 1990, 1991, 1992.

lier studies (Cane et al., 2000; Wu and Lepping, 2002a). The c.c. of $\left|B z_{\min }\right|$ vs. $D_{s t_{\min }}$ is -0.86 for that extended study which means that the long term study apparently gives a better correspondence than the short term studies for this correlation. In addition, Wu and Lepping (2002b) also found that the relationships between storm $D_{s t_{\min }}$ and $V_{\max }$ is poor (i.e., c.c. is -0.58 ), but the correlation for $D_{s t_{\min }}$ vs. $B z_{\min }$ (or $V B s_{\max }$ ) increased dramatically when solar wind speed 
Table 2. Averages of $V_{\max }, D_{s t_{\min }}, B z_{\min }$ and $V B s_{\max }$ for solar quiet and active periods.

\begin{tabular}{lcccc}
\hline & $\begin{array}{c}B z_{\min } \\
(\mathrm{nT})\end{array}$ & $\begin{array}{c}V_{\max } \\
(\mathrm{km} / \mathrm{s})\end{array}$ & $\begin{array}{c}V B s_{\max } \\
(\mathrm{nT})\end{array}$ & $D_{s t_{\min }}$ \\
\hline active period $^{\mathrm{a}}$ & -13 & 522.7 & 6.6 & -100 \\
quiet period $^{\mathrm{b}}$ & -7.9 & 486.8 & 3.3 & -61.9 \\
\hline
\end{tabular}

a Events occurred during the years 1968, 1969, 1979, 1980, 1981, 1990, 1991 and 1992.

b Events occurred during the years 1965, 1966, 1975, 1976, 1985, 1986, 1996, and 1997.

Table 3. The occurrence frequency of MCs which caused a geomagnetic storm during solar quiet and active periods.

\begin{tabular}{ccc}
\hline & quiet & active \\
\hline$D_{s t}>-30 \mathrm{nT}$ & 10 & 7 \\
$-30 \mathrm{nT} \geq D_{s t}>-50 \mathrm{nT}$ & 5 & 5 \\
$-50 \mathrm{nT} \geq D_{s t}>-100 \mathrm{nT}$ & 12 & 7 \\
$D_{s t} \leq-100 \mathrm{nT}$ & 6 & 13 \\
\hline
\end{tabular}

increased. For example, the correlation of $D_{s t_{\min }}$ with $B z_{\mathrm{min}}$ increases from c.c. $=-0.86$ for all events to c.c. $=-0.93$ for events with $V_{\max }>600 \mathrm{~km} / \mathrm{s}$ (Wu and Lepping, 2002b). That implies that solar wind speed also plays a role in the geomagnetic active but not in any simple way.

The result of this study (see Fig. 3) shows clearly that $V_{\max }$ is well correlated with $D_{s t_{\min }}$ in the solar active period (c.c. $=-0.71$ ). In contrast, during the solar quiet period, the correlation is very poor between $V_{\max }$ and $D_{s t_{\min }}$ (c.c. $=-$ 0.19). As expected, we notice that the solar wind speed within MCs on average is higher during the active period. The average of $V_{\max }$ in the solar active period $(522 \mathrm{~km} / \mathrm{s})$ is $\sim 10 \%$ higher than in the solar quiet period $(468 \mathrm{~km} / \mathrm{s})$.

The correlation of $D_{s t_{\text {min }}}$ vs. different solar wind parameters (e.g., $B z_{\min }, V_{\max }$ or $V B s_{\max }$ ) became poor when the Sun became quiet (see Table 1). In addition, the intensity of a storm, in terms of average $D_{s t_{\min }}$ during MC passage was more than $\sim 60 \%$ stronger in the active period $\left(<D_{s t_{\min }}>=-100 \mathrm{nT}\right)$ than in the quiet period $\left(<D_{s t_{\min }}>=-61.9 \mathrm{nT}\right)$. The average strength of $B z_{\min }$ in the active period $\left(<B z_{\min }>=-13 \mathrm{nT}\right)$ is $\sim 60 \%$ greater than in the quiet period $\left(<B z_{\min }>=-7.9 \mathrm{nT}\right)$. The average strength of $V B s_{\max }$ in the active period $\left(<V B s_{\max }>=6.6\right)$ is almost two times greater than that in the quiet period ( $<V B s_{\max }>=3.3$ ). The overall results show that southward IMF is important for causing a geomagnetic storm, as expected, but solar wind speed also plays a role.

Our earlier study showed that the most severe storms caused by MCs/MCLs (i.e., for $D_{s t_{\min }} \leq-100 \mathrm{nT}$ ) during solar cycle 23 occurred in the solar active part of the cycle (Wu et al., 2006). This study shows longer term statistical results (33 years). More than twice as many MCs causing severe geomagnetic storms $\left(D_{s t_{\min }} \leq-100 \mathrm{nT}\right)$ occurred in the solar active period as in the quiet period. The result of this study is consistent with the earlier study by Wu et al. (2006).

\section{Conclusions}

Solar activity plays a major role in the average intensity of geomagnetic storms. Some new results were found as follows, where MC passage is understood in all statements. (1) The intensity of a geomagnetic storm in a solar active period is significantly stronger than in a solar quiet period. (2) The magnitude of negative $B z_{\min }$ is larger in a solar active period than in a quiet period. (3) Solar wind speed in an active period is faster than in a quiet period. (4) $V B s_{\max }$ in an active period is much larger than in a quiet period. (5) Solar wind parameters, $B z_{\min }, V_{\max }$ and $V B s_{\max }$ are well correlated with geomagnetic storm intensity, $D_{s t_{\min }}$, during a solar active period. (6) Solar wind parameters, $B z_{\min }$, and $V B s_{\max }$ are not correlated well (very poorly for $V_{\max }$ ) with geomagnetic storm intensity during a solar quiet period. (7) The speed of the solar wind plays a role in the correlation for solar wind parameters vs. the intensity of a geomagnetic storm. (8) More severe storms with $D_{s t_{\min }} \leq-100 \mathrm{nT}$ caused by MCs occurred in the solar active period than in the solar quiet period.

Acknowledgements. We wish to thank the team at Kyoto University, Kyoto, Japan for providing the $D_{s t}$ data, and the NSSDC at Goddard Space Flight for providing OMNI data. The work of CCW was supported by NASA Grant NNG08GG80G. CCW was also supported in part by the International Collaboration Research Team Program of the Chinese Academy of Sciences.

Topical Editor I. A. Daglis thanks a referee for his/her help in evaluating this paper.

\section{References}

Akasofu, S.-I.: Energy coupling between the solar wind and the magnetosphere, Space Sci. Rev., 28, 121-190, 1981.

Arnoldy, R. L.: Signature in the Interplanetary medium for Substorms, J. Geophys. Res., 76, 5189-5200, 1971.

Bothmer, V. and Rust, D.: The field configuration of magnetic clouds and the solar cycle, in: Coronal Mass Ejections, edited by: Crooker, N., Joselyn, J. A., and Feynman, J., GM 99, Washington, D.C., AGU, 139-146, 1997.

Burlaga, L. F., Sittler, E., Mariani, F., and Schwenn, R.: Magnetic loop behind an interplanetary shock: Voyager, Helios, and IMP 8 observations, J. Geophys. Res., 86, 6673-6684, 1981.

Burton, R. K., McPherron, R. L., and Russell, C. T.: An empirical relationship between interplanetary conditions and Dst, J. Geophys. Res., 80, 4204-4214, 1975. 
Cane, H. V., Richardson, I.G., and St.Cyr, O.C.: Coronal mass ejections, interplanetary ejecta and geomagnetic storms, Geophys. Res. Lett., 27 (21), 3591-3594, 2000.

Chapman, S.: An outline of a theory of magnetic storms, Proc. Roy. Soc. London, A-95, 61-83, 1919.

Doyle, M. A. and Burke, W.: S3-2 measurements of the polar cap potential, J. Geophys. Res., 88, 9125-9133, 1983.

Fairfield, D. H. and Cahill Jr., L. J.: Transition Region magnetic Field and Polar magnetic Disturbances, J. Geophys. Res., 71, 155-169, 1966.

Farrugia, C. J., Burlaga, L. F., and Lepping, R. P.: Magnetic Clouds and the Quiet-Storm effect at Earth, in: Physics of Magnetic Storms, AGU Monograph 98, edited by: Tsurutani, B. T., Gonzalez, W. D. and Kamide, Y., 91-106, 1997.

Gonzalez, W. D., Tsurutani, B. T., Gonzalez, A. L. X., Smith, E. J., Tang, F. and Akasofu, S.-I.: Solar wind-magnetosphere coupling during intense magnetic storms (1978-1979), Planet. Space Sci., 94, 8835-8851, 1989.

Gonzalez, W. D., Joselyn, J. A., Kamide, Y., Kroehl, H. W., Rostoker, G., Tsurutani, B. T., and Vasyliunas, V. M.: What is a geomagnetic storm?, J. Geophys. Res., 99, 5771-5792, 1994.

Kan, J. R. and Lee, L. C.: Energy coupling functions and solar wind magnetosphere dynamo, Geophys. Res. Lett., 6, 577-580, 1979.

Klein, L. W. and Burlaga, L. F.: Interplanetary magnetic clouds at 1 AU, J. Geophys. Res., 87, 613-624, 1982.

Lepping, R. P. and Berdichevsky, D.: Interplanetary magnetic clouds: Sources, properties, modeling, and geomagnetic relationship, in Research Signpost, Recent Res. Dev. Geophys., 7796, 2000.

Lepping, R. P. and Berdichevsky, D.: Eos, Transactions, American Geophysical Union, vol. 82(20), Spring Meeting Suppl., Abstract SH61A-07, p.S332, 2001.

Lepping, R. P., Berdichevsky, D., and Wu, C.-C.: Sun-Earth electrodynamics: The solar wind connection, in Research Signpost, Recent Res. Dev. Astrophys., 1, 139-171, 2003.

Lepping, R. P., Berdichevsky, D. B., Wu, C. C., Szabo, Z., Narock, T., Mariani, F., Lazarus, A. J., and Quivers, A. J.: A summary of WIND magnetic clouds for the years 1995-2003: Model-fitted parameters, associated errors, and classification, Ann. Geophys., 24, 215-245, 2006.
Lepping, R. P., Acuña, M. H., Burlage, L. F., et al.: The wind magnetic field investigation, Space Sci. Rev., 71, 207-229, 1995.

Ogilvie, K. W., Chornay, D. J., Fritzenreiter, R. J., et al.: SWE: A comprehensive plasma instrument for the Wind spacecraft, Space Sci. Rev., 71, 55-77, 1995.

Perreault, W. K. and Akasofu, S.-I.: A study of geomagnetic storms, Geophys. J. R. Astron. Soc., 54, 547-573, 1978.

Tsurutani, B. T., Smith, E. Gonzalez, W. D., Tang, F., and Akasofu, S. I.: Origin of interplanetary southward magnetic fields responsible for major magnetic storms near solar maximum (19781979), J. Geophys. Res., 93, 8519-8531, 1988.

Wilson, R. M.: On the behavior of the Dst geomagnetic index in the vicinity of magnetic cloud passages at Earth, J. Geophys. Res. 95, 215-219, 1990.

Wu, C.-C. and Lepping, R. P.: The effects of magnetic clouds on the occurrences of geomagnetic storms: the first four years of WIND, J. Geophys. Res., 107 (A10), 1314, doi:10.1029/2001JA000161, 2002a.

Wu, C.-C. and Lepping, R. P.: The effect of solar wind velocity on magnetic cloud-associated magnetic storm intensity, J. Geophys. Res., 107 (A11), 1346, doi:10.1029/2002JA009396, 2002 b.

Wu, C.-C. and Lepping, R. P.: Relationships for predicting magnetic clouds related geomagnetic storm intensity, J. Atmos. Terr. Phys., Vol 67/3, 283-291, doi:10.1016/j.jastp.2004.07.040, 2005.

Wu, C.-C., Lepping, R. P. and Gopalswamy, N.: Variations of magnetic clouds and CMEs with solar activity cycle, in Proceedings of International Solar Cycle Studies Symposium 2003: Solar variability as an input to the Earth's environment, SP535, Tatranska Lomnica, Slovak Republic, June 23-28, 429-432, 2003.

Wu, C.-C., Lepping, R. P., and Gopalswamy, N.: Relationships among magnetic clouds, CMEs and geomagnetic storms, Solar Phys., in press, 2006. 